Article

\title{
Game Theoretic Spectrum Allocation in Femtocell Networks for Smart Electric Distribution Grids
}

\author{
Ali Mohammadi ${ }^{1, *}$, Mohammad Javad Dehghani ${ }^{2,3}$ (iD) and Elham Ghazizadeh 4 \\ 1 Department of Electrical and Computer Engineering, Louisiana State University, Baton Rouge, \\ LA 70803, USA \\ 2 Department of Electrical and Electronic Eng, Shiraz University of Technology, Shiraz 71557-13876, Iran; \\ Dehghani@sutech.ac.ir \\ 3 Regional Information Center for Science and Technology, Shiraz 71946-94171, Iran \\ 4 Department of Electrical and Systems Engineering, Washington University, St. Louis, MO 63130, USA; \\ elham@wustl.edu \\ * Correspondence: amoha39@1su.edu; Tel.: +1-225-394-5763
}

Received: 24 May 2018; Accepted: 18 June 2018; Published: 22 June 2018

\begin{abstract}
Ever growing penetration of the behind-the-meter technologies is changing the electricity consumption profiles of end-users. Intelligent coordination of these emerging technologies through a robust communication infrastructure enables their seamless integration with electric utilities' operation. In this context, an efficient and reliable communication infrastructure plays a pivotal role in enabling optimal integration of emerging resources. In this paper, we propose a game-theory based method to enhance efficiency of the underlying communication network. Specifically, we focus on Femtocell communication technology which is one the promising options for improving poor indoor communication coverage. The major drawback for using femtocell communication technology is cross-layer interference of femto users (FUs) and macro users (MUs) which adversely impact network performance. In this paper, we propose a novel approach for sharing spectrum in a cognitive radio system with FUs and MUs as primary and secondary users, respectively. The underlying problem is formulated as Stackelberg game that is joined with a convex optimization problem. In this study, MUs and FUs are assumed to be selfish, rational and motivated to achieve maximum utility function, while MUs are competing to obtain maximum bandwidth. Finally, we present a closed form solution for the proposed approach which obtains a unique Nash Equilibrium and prioritizes the access of MUs to femto-base stations. Simulation results provide proof of concept and verify the effectiveness of our mathematical modeling.
\end{abstract}

Keywords: smart grid; femtocell; macro; bandwidth allocation; Stackelberg game; convex optimization; hybrid access; interference

\section{Introduction}

Ever increasing growth of behind-the-meter Internet of Things (IoT) devices are increasing the overhead of communication networks. Emergence of these new technologies is also affecting the electricity consumption behavior of end-users, hence, influencing operation of the electric grid [1-4]. Intelligent communication and coordination of these behind-the-meter assets (e.g., battery energy storage and solar photovoltaics) is essential for their integration with the operation of the electric network, improving resiliency of the power grid in the face of unexpected interruptions, and paving the way toward smarter and cleaner electric distribution networks [2,5]. Further, the emerging concept of smart grids introduces a new class of complexities in terms of interdependencies among power systems, communication networks, and electrified transportation networks [3,6]. The interdependent 
operation of power and transportation networks is thoroughly investigated in $[1,7,8]$ by proposing an all-inclusive framework that takes the constraints of both networks into account. According to [2,7], an efficient and reliable communication platform plays a pivotal role in a cost-effective roadmap towards electrified transportation networks while meeting the limits of the power systems. In addition to the mentioned roles of communication networks, they are an inevitable part of distributed and parallel computing platforms. According to [9-12], state-of-the-art algorithms to solve the complex optimization problems in energy systems requires a connected communication graph to make sure all agents are communicating in a real-time fashion.

Femtocell technology is the best way to improve indoor communication networks [13]. It can improve the coverage condition and capacity of cellular network that connects behind-the-meter assets. Femtocell communication is a cost-effective technology that requires low power and provides a satisfactory quality of service (QoS) for its users in short range of coverage. Femtocells are connected to the cellular network through the cable, Ethernet and so on. Macro Users (MU)s (a macrocell is a cellular network that consist of large towers with high amount of power and large range of coverage; the users that are connected to macrocells are called macro users (MUs). We explain the details of MUs in Section 2) that are located near femto-base stations (FBS)s may suffer from intense interference. This results in MUs tendency to connect to more desirable FBSs instead of leaving the network. On the other hand, macro base stations (MBS)s prefer MUs to utilize low-cost traffic FBSs rather than expensive ones [14]. Another factor that encourages users to leverage femtocell technology is a better performance due to high data rate of $3 \mathrm{G}$ network compared with Wi-Fi technology [14]. Although femtocell technology has many benefits for users and network, this technology has yet to address problems such as usage organization, usage optimization and inter-tier and intra-tier interference issues. These pressing challenges highlight the need for an accurate resource allocation technique.

Since FBSs can be installed according to the owner preferences, they can be located close to each other or far. Co-located FBSs which worked in same frequency and time slot may face interference when their coverage ranges overlap. There are two kinds of femtocell interferences: First, cross-tier which occurs when a source and victim users work on different tiers. For example, an undesirable signal that comes from MBSs leads to cross-tier interference. In literature, this interference problem has been tackled from different angles including power [15] and spectrum allocations [16-18] in open or closed access setups $[19,20]$. The second type of femtocell interference is co-tier interference which happens when the source and victim work in the same tier. For instance, two femtocells placed in adjacent neighborhoods can interfere with each other. Co-tier interference is a difficult problem to cope with [21,22]. Prior studies have focused on mitigating the interference and improving the spectral efficiency [23,24].

Due to the interference effect on network performance, choosing a proper access mechanism based on the degree of interference and the distance among user is required. The access mechanisms are categorized into three major groups: closed access, open access, and hybrid access. In the closed access only registered femto users can connect with FBS, i.e., femto users (femto users (FUs) are registered the femto base station users. We explain the details of FUs in Section 2) (FUs) are satisfied with their service. Although FUs have exclusive access to all sources, they may suffer from high interference caused by macro users who are located far from MBS but near FBS, i.e., dead zones, because the macro users may work in the same frequency as FUs. The second access mechanism is referred to as open access. In contrast with the closed access setup, this system allows all wireless users to connect to FBS and mitigates dead zones. Since in this accessibility there is no difference between FUs and MUs, FUs may face traffic congestion and not be able to attain a desirable rate. Hybrid access, the third class of access mechanism, inherits advantages of closed and open access mechanisms, i.e., it allows all macro users to connect with FBSs and guarantees a desirable performance and rate for FUs. In the hybrid access, MUs can access FBS resources under a certain circumstance. For example, MUs can get resources, but they should pay instead. In contrast with closed and open access setups, hybrid access can reduce cross-tier interference and enhance FU performance reliability $[25,26]$. Hence, this access 
mechanism enhances network capacity. In this work, the merit of hybrid access in comparison to other accesses is investigated by developing an intelligent resource allocation.

In this paper, we propose a novel spectrum allocation approach to share access between FUs and MUs in a hybrid access setup. FUs and MUs are presumably rational and selfish. FBSs are willing to open certain spectrum, in a hybrid mode, to enhance the overall network capacity. In some cases, however, FBSs prefer to select closed access. As a result, only their registered FUs can have access to resource [27]. FBSs are responsible for determining bandwidth assignment to MUs and FUs. In this regard, the monetary value of FBS services directly impact these assignments and operation of neighboring FUs and MUs. By increasing the resource allocated to femto users, FBSs can enhance FUs' performance but at a higher cost. Consequently, access to more resources inflates expenses for MUs. Given these properties, this resource allocation problem naturally lends itself to a game theory setup that determines optimal payment flow among stakeholders, i.e., MUs, FUs, and FBS, as well as optimal resource allocation according to service expenses.

With growing the number of users in a network, finding an optimal resource allocation is challenging. To tackle the resource allocation problem, game theory becomes a well-known method to model and analysis. In the telecommunication problems, usually, a Stackelberg game is used to assign the rate and power to users. The Stackelberg game is a strategic game, in which two types of players participate, leaders and follower. The leaders select the strategy of game and act first, while the followers follow the rule of game. Leaders know that each decision has the specific consequence on the followers' decision and based on the game rule followers should follow the leaders' actions. Some of the interesting works on resource allocation is as follows. The power allocation for two-tier networks is modeled in $[28,29]$ while the problem is solved by robust Stackelberg game [28,30]. In order to assume interference constraints for uplink resource allocation problem, a Bayesian Stackelberg game is proposed in $[14,23,31,32]$. In $[14,31]$ price-based resource allocation is presented while the macrocell protection is considered.

In this paper, we pose the aforementioned resource allocation problem as a Stackelberg game theory problem. In order to highlight the contribution of our work, we count them as follows:

- Since the overall bandwidth is limited, the total bandwidth that is divided between MUs and FUs should meet bandwidth capacity limitation. To this end, at first, we formulate a game theory and convex optimization problem. Then we derive a closed form solution for the proposed formulation. To tackle this problem, authors in [33] solve a resource allocation problems in an iterative fashion whereas [34] propose a Fuzzy Logic approach to solve this problem.

- In this work, we offer an efficient, robust spectrum assignment technique, through two adjustable coefficients, the first coefficient is used to specify the allocation strategy. To this end four strategies are considered: maximizing femtocell utility, establishing fair access for all MUs, removing macro users with maximum bandwidth needs while promoting macro users with bandwidth minimum needs and vice-versa.

- The second coefficient prioritizes each MU to access more or less amount of bandwidth. The coefficient can be calculated based on the distance, signal-to-interference-plus-noise ratio (SINR) or similar index. For example, since the distance between FBS and MUs increase, FBS usually spend more power to transmit a signal. In this case, FBS prefers to assign less priority to such a user.

The rest of paper is organized as follows: the system model is described in Section 2, and the problem formulation is elaborated in Section 3. In Section 4, game theory analysis is presented, while the Stackelberg game and Nash Equilibrium are presented in Section 5. The results are presented in Section 6, and the last section concludes the paper. 


\section{System Model}

In this section, the proposed system and network model are elaborately described. We consider the downlink state of a two-tier network which consists of uniformly distributed femtocells in one macro-cell area. The macro-cell and femtocells coverage radius values are $R_{M}$ and $R_{F}$, respectively $\left(R_{M} \gg R_{F}\right)$. Further, total transmit power for MBS and FBSs are $P_{M}$ and $P_{F}$, respectively $\left(P_{M}>>P_{F}\right)$. Assume that each FBS has one femto user (FU) and $k$ macro users (MUs) which are distributed uniformly near each FBS and are located its coverage radius. All FBSs operate in hybrid access mode, i.e., MUs can use FBS spectrum under certain conditions. Further, any MU suffering from high interference due to closeness to FBS is taken into account. In this paper, due to the negative effect of FBSs' range overlap on FUs performance, FBSs are placed far from each other; hence, interference between them is insignificant and co-tier interference is neglected. Cross-tier interference affects SINR of each MU, and this parameter is a proper index to specify which macro user needs less power to reach its desired SINR; therefore, a MU with higher SINR gets higher priority. In this regard, FBSs assign more bandwidth to MUs with higher priority because this leads to a decrease in overall consumable power.

In this paper, FU requires a bandwidth equal to $w_{f}$. Additionally, each macro user (MU) needs a bandwidth of $w_{i}$ and total bandwidth of FBS is $w$. In this regard, total bandwidth that FU and all MUs need is larger than that of the total bandwidth assumed which means:

$$
w_{f}+\sum_{i=1}^{k} w_{i}>w
$$

Since the total requested bandwidth is larger than the available bandwidth, there is a competition between FU and each MU form. In order to tackle this problem, we propose to develop a Stackelberg game-based formulation. In the proposed game theoretic framework, each FBS splits $w$ between FU and MUs. Let $w_{f}^{*}=b w$ represent the bandwidth allocated to FU and $\sum_{i=1}^{k} w_{i}^{*}=(1-b) w$ demonstrates the bandwidth which is allocated to all MUs where $b$ is the bandwidth allocation ratio and is expressed as $b=\frac{\alpha^{*} w_{f}}{w} ; \alpha^{*}$ denotes the satisfaction percentage of femto user. In order to have a proper hybrid mechanism, the system should consider $0<\alpha^{*}<1$. If $\alpha^{*}$ becomes greater than one, it means system assigns more bandwidth than the requirement of femto users. Our focus is on analyzing the downlink and uplink states in a similar way. To this end, in this paper, we design a Stackelberg game theory which allocates bandwidth by prioritizing MUs. In order to achieve optimal assignment of bandwidth, we propose to formulate and solve a joint convex optimization and game theory problem.

\section{Problem Formulation}

In this section, spectrum sharing in hybrid access mode is proposed in which MUs are desired to motivate FBSs to share their spectrum through a refunding scheme. In hybrid access mechanism, MUs are satisfied and overall network capacity increases because FBSs can enhance their utilities by compensating service expenses and MUs obtain bandwidth. Therefore, this is a win-win game, and the aim of our study is to assign high data rate for FUs while providing a better condition for MU to get rid of high interference. To achieve this goal, this problem is formulated as a Stackelberg game.

\subsection{Utility Function}

Utility function of FBSs as given in (2) consists of two parts in which, the first term is the amount of spectrum which is assigned to FUs, and the second part represents the revenue which MUs pay [35]:

$$
U_{F B S}=\Omega_{F} U_{w}+\sum_{i=i}^{k} m_{i},
$$


where $U_{w}$ is the churn rate of femto user, $\Omega_{F}$ is the equivalent revenue and $m_{i}$ is the revenue that each macro user pays. When $U_{w}$ is decreased by one percent, $U_{w}$ is the data rate utility which demonstrates FU's satisfaction and is defined as a sigmoid function [35]. Sigmoid function is one of the best functions for approximating user satisfaction with respect to resource allocation [36] and is given by:

$$
U_{w}=\frac{1}{1+e^{-a\left(\alpha w_{f}-w_{0}\right)}}
$$

Here, $a$ represents the femto user's sensitivity and $w_{0}$ is the bandwidth reservation of FU. In addition, $\alpha$ is the femto user's satisfaction factor, $w_{f}$ is the desirable bandwidth of femto user and $\alpha w_{f}$ represents bandwidth that is allocated to FU.

Hence, the objective of FU optimization problem is to maximize:

$$
\max U_{F B S}
$$

Moreover, macro users' utility function consists of two terms; the bandwidth that MUs have attained with a reasonable payment and the refunding that each macro user should pay:

$$
U_{i}=\Omega_{m} w_{i}^{*}-m_{i}
$$

Here, $w_{i}^{*}$ can be derived as:

$$
w_{i}^{*}=\frac{\mu\left(w-\alpha w_{f}\right)\left(m_{i} w_{i} Z_{i}\right)}{\sum_{i=1}^{k} w_{i} Z_{i} \cdot \sum_{i=1}^{k} m_{i}}
$$

$\Omega_{m}$ denotes the equivalent revenue per unit bandwidth that macro user receives from FBS and $w_{i}^{*}$ is the allocated bandwidth to the $i$-th MU. Moreover, $\mu$ is a parameter to regulate assigned bandwidth and revenue and can establish fairness among MUs or it can maximize total revenue of FBS. Also, $Z_{i}$ specifies the priority of each MU and depends on each macro user's specification (e.g., SINR). In fact, this parameter increases MU bandwidth assignment.

Since the FBS wants to assign the whole bandwidth $(w)$ to femto and macro users, utility function described by (5) should be maximized under the condition which leads to bandwidth allocation to FU and MUs as in [33]:

$$
\begin{gathered}
\max U_{i} \\
\text { subject to } w_{f}^{*}+\sum_{i=1}^{k} w_{i}^{*}=w \\
w_{i}^{*}<w_{i}
\end{gathered}
$$

By substituting (5) and (6) in (7), these conditions can be derived as:

$$
\begin{gathered}
\left(w-\alpha w_{f}\right)\left[\sum_{i=1}^{k} w_{i} Z_{i} \cdot \sum_{i=1}^{k} m_{i}-\mu \sum_{i=1}^{k} w_{i} Z_{i} m_{i}\right]=0, \\
\sum_{j=1}^{k} w_{j} Z_{j} \sum_{j=1}^{k} m_{j}-\left(w-\alpha w_{f}\right) m_{j} Z_{j} \geq 0 .
\end{gathered}
$$

\section{Game Theory Analysis}

In this section, the problem of bandwidth allocation is formulated as a non-cooperative Stackelberg game, in which FU is considered as a leader or primary user, and MUs act as followers or secondary users. In this section, we analytically proved that the aforementioned problem has unique Nash Equilibrium by which the utility of both FU and MUs are maximized. 
Since both FU and MUs are rational as well as selfish, and they intend to maximize their utility, game theory is one of the best tools for solving this problem. In this paper, it is assumed that both FU and MUs prefer to work in hybrid access mode where both can maximize their utility. Therefore, it is reasonable to formulate the problem based on Stackelberg game theory.

The Stackelberg game is a strategic game that consists of two types of player: leaders and follower. Leaders select a strategy and act, while the followers will choose their strategy based on leaders' action. Since the leaders know the follower reaction, they select the best strategy that maximizes their benefits. In this paper the Stackelberg game proceeds in two steps. First, an FBS specifies the amount of bandwidth that intends to share with MUs while it is aware that its move will not only be seen by MUs, but also it will influence the decision of MUs. Thus, FBS determines optimum $\alpha$ to maximize its utility. In the next step, followers should obtain the leader's strategy, therefore, MUs specify their revenue to maximize their utility while considering FBS determination. This decision affects the other side of the game. So, the solution procedure can be described as follows:

- Find the optimal value for revenue $(m)$ and Lagrange multipliers based on $\alpha$.

- Derive the condition that $U_{F B S}$ can be a maximized point.

- $\quad$ Find an optimal value for $\alpha$ by solving first order optimality solutions.

\subsection{Macro Users' Payment}

To solve the resource allocation problem, according to Stackelberg game theory method, a backward induction method is used [35]. First, the problem of having one unique Nash Equilibrium point is indicated where MUs have maximum utility. The problem described by (7) is a convex optimization problem. After solving this problem, FBS is able to specify the optimum value of $\alpha$ to maximize its utility. In order to solve this problem, we first prove that (7) is a convex.

Afterward, we find the optimal solution by solving first-order optimality conditions of underlying problem [33]. To this end, the Lagrangian of MUs utility can be derived as:

$$
\begin{gathered}
L\left(\alpha, m_{i}, \lambda_{0}, \lambda_{i}\right)=\Omega_{m} \mu \frac{\left(w-\alpha w_{f}\right)\left(m_{i} w_{i} Z_{i}\right)}{\sum_{j=1}^{k} w_{j} Z_{j} \cdot \sum_{j=1}^{k} m_{j}}-m_{i}+ \\
\lambda_{0}\left(w-\alpha w_{f}\right)\left[\sum_{j=1}^{k} w_{j} Z_{j} \cdot \sum_{j=1}^{k} m_{j}-\mu \sum_{j=1}^{k} w_{j} Z_{j} m_{j}\right]+ \\
\sum_{j=1}^{k} \lambda_{j} \cdot\left[\sum_{j=1}^{k} w_{j} Z_{j}-\left(w-\alpha w_{f}\right) Z_{j}\right]
\end{gathered}
$$

where $\lambda_{0}$ and $\lambda_{j}$ are the Lagrange multiplier. Based on KKT condition, $\lambda_{j}$ should be equal to 0 . To prove the convexity of macro users' utility in Lagrangian form, the second derivative on mi is calculated as:

$$
\begin{gathered}
\frac{\partial L\left(\alpha, m_{i}, \lambda_{0}\right)}{\partial m_{i}}=\Omega_{m} \mu \frac{\left(w-\alpha w_{f}\right) \sum_{i \neq j}^{k} m_{j}}{\sum_{j=1}^{k} w_{j} Z_{j} \cdot\left(\sum_{j=1}^{k} m_{j}\right)^{2}} w_{i} Z_{i}-1+ \\
\lambda_{0}\left(w-\alpha w_{f}\right)\left[\sum_{j=1}^{k} w_{j} Z_{j}-\mu w_{i} Z_{i}\right] \\
\frac{\partial^{2} L\left(\alpha, m_{i}, \lambda_{0}\right)}{\partial m_{i}^{2}}=-2 \Omega_{m} \mu \frac{\left(w-\alpha w_{f}\right) \sum_{i \neq j}^{k} m_{j}}{\sum_{j=1}^{k} w_{j} Z_{j} \cdot\left(\sum_{j=1}^{k} m_{j}\right)^{3}} w_{i} Z_{i}<0 .
\end{gathered}
$$

The second order derivative of $L$ with respect to $m_{i}$ is always negative, so the problem in (10) is convex; hence, this function has a maximum. In order to solve the convex optimization problem, $m_{i}^{*}$ should be obtained under Karush-Kuhn-Tucker (KKT) condition. With regard to this, each MU 
tries to achieve Nash Equilibrium because the best answer can maximize its utility, consequently, MUs compete with each other. Therefore, $m_{i}^{*}$ is given by:

$$
\begin{gathered}
m_{i}^{*}=\frac{\left(A X-Y X^{2}\right)}{A}+\frac{B X^{2}}{A \mu w_{i} Z_{i}} \\
X=\frac{(k-1) A}{k Y-B(D / \mu)}
\end{gathered}
$$

In which:

$$
\begin{gathered}
A=\Omega_{m} \frac{w-\alpha w_{f}}{w_{x}} \\
B=-1+\lambda_{0}\left(w-\alpha w_{f}\right) w_{x} \\
Y=\lambda_{0}\left(w-\alpha w_{f}\right) \\
D=\sum_{j=1}^{k} \frac{1}{w_{j} Z_{j}} \\
w_{x}=\sum_{j=1}^{k} w_{j} Z_{j} \\
X=\sum_{i=1}^{k} m_{i} .
\end{gathered}
$$

Because $B$ is a dependent variable with respect to $\lambda_{0}, X$ and $m_{i}^{*}$ also depend on $\lambda_{0}, \lambda_{0}$ can be obtained from the above condition as:

$$
\lambda_{0}=\frac{D w_{x}-\mu\left(k-k^{2}+D w_{x}\right)}{\left(w-\alpha w_{f}\right) w_{x}\left(\mu^{2}+D w_{x}+\mu\left(-2 k+k^{2}-D w_{x}\right)\right)} .
$$

Appendix A provides detailed analytical discussion for derivation of (21).

By putting $\lambda_{0}$ in $B, X$ is obtained as follows:

$$
X=\Omega_{m} \frac{\left(w-\alpha w_{f}\right)\left(\mu^{2}-2 \mu k+\mu k^{2}+D w_{x}-\mu D w_{x}\right)}{\mu\left(k^{2}-D w_{x}\right)},
$$

where $X$ demonstrates overall revenue that FBS can get from MUs. Also, the value of parameter $\mu$ has a vital role in the allocation and price. This parameter both affects the sign of $m_{i}^{*}$ and determines the strategy of allocation. At first, the condition that $\mu$ is acceptable should be obtained, then, how $\mu$ determines a strategy should be specified as well as each bandwidth assigning strategy. The condition that leads to turning the sign of $m_{i}^{*}$ to a positive state is as follows:

$$
\begin{aligned}
& \max \left\{\frac{\left(w_{i} Z_{i} D-k\right) w_{x}}{k w_{i} Z_{i}-w_{x}},\right. \\
& \left.\frac{D w_{x}{ }^{2}+2 k w_{x}-k^{2} w_{x}-\sqrt{-4 D w_{x}{ }^{3}+\left(-D w_{x}{ }^{2}-2 k w_{x}+k^{2} w_{x}\right)^{2}}}{2 w_{x}}\right\}< \\
& \mu<\min \left\{\frac{\left(w_{i} Z_{i} D-k\right) w_{x}}{k w_{i} Z_{i}-w_{x}},\right. \\
& \left.\frac{D w_{x}{ }^{2}+2 k w_{x}-k^{2} w_{x}+\sqrt{-4 D w_{x}{ }^{3}+\left(-D w_{x}{ }^{2}-2 k w_{x}+k^{2} w_{x}\right)^{2}}}{2 w_{x}}\right\}
\end{aligned}
$$

Based on the above condition, two proposed values for parameter $\mu$ are placed in this range. If $\mu=k$ is selected, the system can establish fairness among MUs. In this case, the ratio of assigned 
bandwidth to desired bandwidth is directly dependent on $Z_{i}$, furthermore, all macro users pay the same revenue if the priority factor $Z_{i}$ is assumed to be one, i.e.,:

$$
\frac{w_{i}^{*}}{w_{i}} \propto Z_{i}
$$

On the other hand, if $\mu=\sqrt{D w_{x}}$, not only is the whole revenue, that FBS can get, maximized but also the utility of FBS reaches its maximum. Two other strategies are defined to compete with these proposed methods. The $\mu_{\text {low }}$ is considered as the lower bound and $\mu_{u p}$ is the upper bound. If $Z_{i}$ is assumed equal to one, the system by selecting $\mu_{\text {low }}$ deprives one of the macro users which needs minimum desired bandwidth from allocation, and the system promotes macro users requiring higher desired bandwidth. Also, choosing $\mu_{u p}$ removes MU with maximum desired bandwidth and promotes the macro user which needs less; this is not rational and useful for the system. In this case, if the allocated bandwidth is more than the desired bandwidth, system adjusts the assigned bandwidth to the desired one. Considering the analytical results of MUs payment, FBS can maximize its utility function based on revenue which was obtained from (22). A leader knows that its strategy will influence the follower's decision, so it specifies $\alpha^{*}$ which maximizes its utility. Substituting (22) with (2), the utility function of FBS is expressed as:

$$
U_{F B S}=\Omega_{f}\left(\frac{1}{1+e^{-a\left(\alpha w_{f}-w_{0}\right)}}\right)+\left(\frac{w}{w_{f}}-\alpha\right) \eta,
$$

where $\eta=\Omega_{m} \frac{w_{f}\left(\mu^{2}-2 \mu k+\mu k^{2}+D w_{x}-\mu D w_{x}\right)}{\mu\left(k^{2}-D w_{x}\right)}$.

In order to find the optimum value of $\alpha^{*}$, first, the utility function of FBS that has a maximum point should be proved. Next, based on the first derivative, the maximum point will be obtained. The first and second derivatives of UFBS with respect to $\alpha$ are calculated as:

$$
\begin{gathered}
\frac{\partial U_{F B S}}{\partial U \alpha}=\frac{a \Omega_{f} w_{f} e^{-a\left(\alpha \mathrm{w}_{f}-\mathrm{w}_{0}\right)}}{\left(1+e^{-a\left(\alpha \mathrm{w}_{f}-\mathrm{w}_{0}\right)}\right)^{2}}-\eta, \\
\frac{\partial^{2} U_{F B S}}{\partial \alpha^{2}}=\Omega_{f} \frac{a^{2} \mathrm{e}^{-a\left(\alpha \mathrm{w}_{f}-\mathrm{w}_{0}\right)}\left(-1+\mathrm{e}^{-a\left(\alpha \mathrm{w}_{f}-\mathrm{w}_{0}\right)}\right) w_{f}^{2}}{\left(1+\mathrm{e}^{-a\left(\alpha \mathrm{w}_{f}-\mathrm{w}_{0}\right)}\right)^{3}} .
\end{gathered}
$$

When $\alpha>\frac{w_{0}}{w_{f}}$, the second derivative of UFBS with respect to $\alpha$, is always negative, UFBS is concave in terms of $\alpha$, so it has a maximum point. On the other side, when $\alpha<\frac{w_{0}}{w_{f}}$, the second derivative of UFBS with respect to $\alpha$, is always positive, UFBS has a minimum. To further clarify the point, it is from the second derivative that the function falls down at first, then, it approaches the minimum point. Next, it goes up and reaches the maximum point of UFBS in $\alpha^{*}$ and the function decreases again.

When the following conditions are satisfied:

$$
\begin{gathered}
\eta \leq \frac{a \Omega_{f} w_{f}}{4} \\
\Omega_{f} \geq \eta \frac{w_{f}}{w_{f}-1} \alpha^{*}
\end{gathered}
$$


the optimum $\alpha^{*}$ by assigning the first derivative of UFBS to 0 can be found. In this case, there are a local minimum point $\alpha_{1}$ and a maximum point $\alpha_{2}$ which are obtained as follows:

$$
\begin{aligned}
& \alpha_{1}=\frac{a w_{0}-\ln \left(\frac{a \Omega_{f} w_{f}-2 \eta+\sqrt{\left(a \Omega_{f} w_{f}\right)^{2}-4 \eta * a \Omega_{f} w_{f}}}{2 \eta}\right)}{a w_{f}} \\
& \alpha_{2}=\frac{a w_{0}-\ln \left(\frac{a \Omega_{f} w_{f}-2 \eta-\sqrt{\left(a \Omega_{f} w_{f}\right)^{2}-4 \eta * a \Omega_{f} w_{f}}}{2 \eta}\right)}{a w_{f}}
\end{aligned}
$$

The optimal value should have two features: first, $\alpha^{*}$ should satisfy criteria which are presented in (32) and the other feature is that $\alpha^{*}$ should be a global maximum point in this criterion, so the optimum value should be on the interior range of domain:

$$
0 \leq \alpha_{2} \leq 1
$$

The local maximum $\alpha_{2}$ should be a global maximum which is expressed as:

$$
U_{F B S}(0)<U_{F B S}\left(\alpha_{2}\right)
$$

When $\omega_{f} \geq \eta \frac{w_{f}}{w_{f}-1}$, it can be easily proved that $\alpha_{2}$ is the global maximum and $U_{F B S}^{*}=U_{F B S}\left(\alpha_{2}\right)$.

When $\eta=\frac{a \Omega_{f} w_{f}}{4}$, MUs provide maximum revenue for FBS. In this case, MUs motivate FBS to share more bandwidth and decrease $\alpha^{*}$. By substituting this value into (28), $\alpha^{*}$ is obtained as $\alpha^{*}=\frac{w_{0}}{w_{f}}$, so this is the minimum value of $\alpha^{*}$ if and only if $U_{F B S}(0)<U_{F B S}\left(\alpha_{2}\right)$.

If $\alpha^{*}=0$, it means that FBS prefers to assign the whole bandwidth to MUs only and if $\alpha^{*}=1$, the system allocates the desired bandwidth that FU desires, so the satisfaction parameter is equal to 1 . The system does not assign more than the desired bandwidth to user. This causes the maximum value of $\alpha^{*}$ to be less than 1. In other words, the system always works in hybrid access mode because it is not reasonable for the system to allocate greater bandwidth than the desired bandwidth to FU, i.e., closed access.

\section{Simulation Results and Discussion}

In this section, a system model which consists of one MBS and FBS is considered. In this case, it is assumed that one FU which is known as a home user is connected to FBS which is surrounded by $\mathrm{k}$ macro users. Also, MUs which are placed adjacent to FBS prefer to be connected to FBS instead of MBS; hence, the system is assumed to be in hybrid access mode, and the total available bandwidth is $50 \mathrm{MHz}$. For better results, Monte Carlo simulation is used, and all results are taken on average of 1000 times run. The simulation follows LTE specification. In order to clarify more how the game problem is solved backward, the solution procedure is demonstrated in Figure 1. In this flowchart, we should initialize the tuning parameters. Then the strategy index $(\mu)$ should be specified between the acceptable range (i.e., based on (23)). In the next step, both $m$ and $\lambda$, should be calculated based on $\alpha$. Since in the utility function of FBS all parameters are plugged in based on $\alpha$. We can derive an optimal point by using the first derivative of it. Finally, when $\alpha^{*}$ obtained, other variables can be calculated.

This paper could find specific $\lambda_{0}$ compared to [33], so it does not require to be iterative in order to converge to the best solution, and bandwidth allocation is done within a scheduled time unit. Moreover, we prove that the whole bandwidth which is assigned to MUs and FU is equal to whole FBS bandwidth. This is because of convex optimization used in the Stackelberg game. The allocated bandwidth depends on both the cost that MUs pay and the priority factor. Depending on the $Z_{i}$ of each MU, each user that has a higher priority factor has a better condition and gets more bandwidth. In this case, parameter $\mu$ 
determines the main strategy to assign bandwidth, and changing this parameter specifies how the system distributes the bandwidth among MUs while following the constraint expressed in (7).

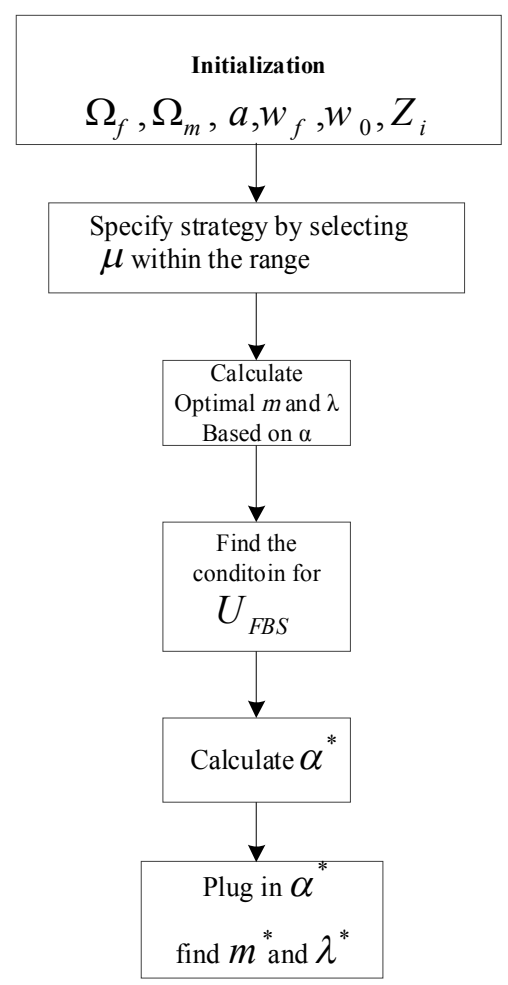

Figure 1. Solution procedure.

The parameter $\mu$ has an important role in determining the bandwidth allocation and four $\mu$ s are defined: $\mu_{o p t}, \mu_{\text {fair }}, \mu_{\text {low }}$ and $\mu_{u p}$. In these terms, $\mu_{\text {opt }}$ and $\mu_{\text {fair }}$ are always placed between $\mu_{\text {low }}$ and $\mu_{u p}$. As mentioned above, $\mu_{\text {opt }}$ means that if $\mu=\mu_{\text {opt }}$, the system can obtain maximum revenue and this is the optimum value for FBS to maximize its utility. In this case, MUs which require less bandwidth suffer from more payment but obtain more reception to desired ratio of bandwidth. On the other hand, by choosing $\mu_{\text {fair }}$ the system allocates its bandwidth based on MU merit which is expressed by $Z_{i}$ and if $Z_{i}=1$ for all MUs, the ratio of the allocated bandwidth to the desired one is equal for all. By doing so, the system can share its bandwidth fairly between all MUs. Choosing $\mu_{\text {low }}$ and $\mu_{\text {up }}$ leads to MUs with minimum and maximum of $Z_{i} w_{i}$ to be removed from allocation, respectively.

\subsection{Impact Number of Macro Users on System Performance with the Same Priority}

In this section, $k$ MUs are placed near FBS and their desired bandwidths are uniformly distributed between 12 and 18 . The equivalent revenue parameter $\Omega_{s}$ is considered one.

The proposed Stackelberg game theory with different $\mu$ s is evaluated. Figure 2a,b depict the utility of FBS and the revenue which is based on the number of MUs. It is demonstrated that the system obtains maximum revenue and utility of FBS by choosing $\mu_{\text {opt }}$; also, choosing $\mu_{\text {fair }}$ causes the system performance to be optimum, so by choosing $\mu_{\text {fair }}$ and $\mu_{\text {opt }}$, FBS utilities and revenues have an insignificant difference. Moreover, the system decreases the revenue and FBS utility by choosing $\mu_{\text {low }}$ and $\mu_{u p}$ compared to two other methods, but it raises the average utility of MU. Although choosing $\mu_{\text {low }}$ and $\mu_{u p}$ increases the average utility of macro user, choosing $\mu_{\text {low }}$ causes to remove MU with minimum bandwidth and $\mu_{u p}$ removes the maximum one. 


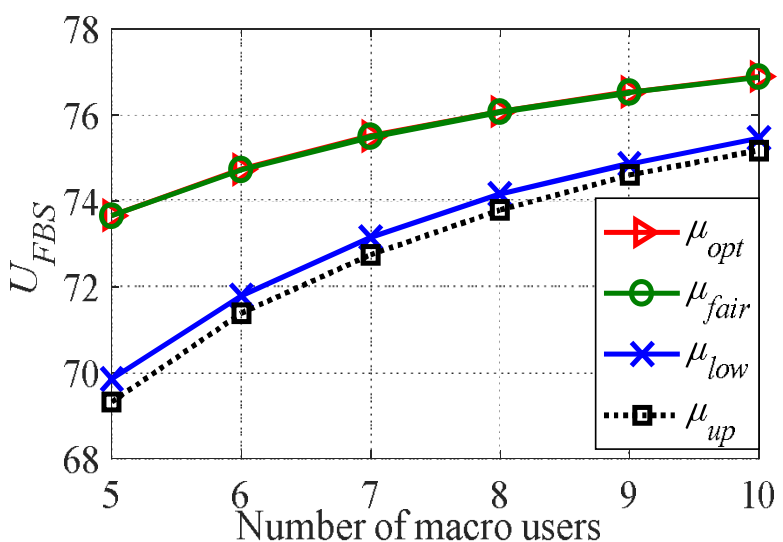

(a)

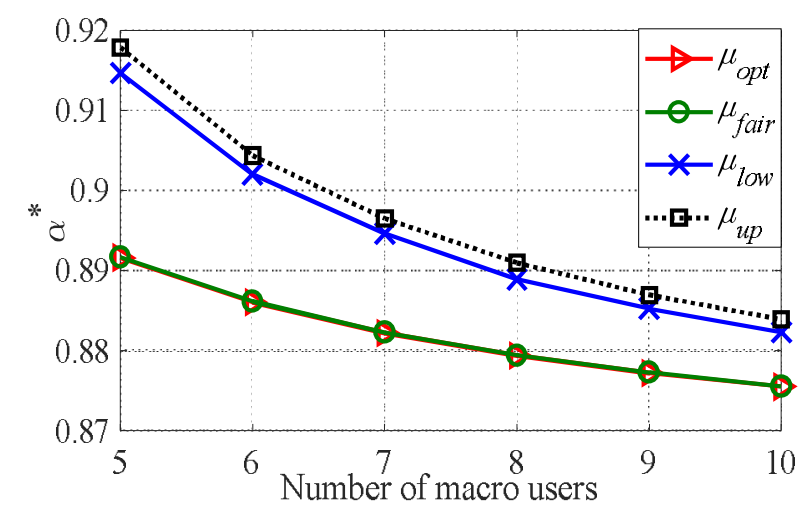

(c)

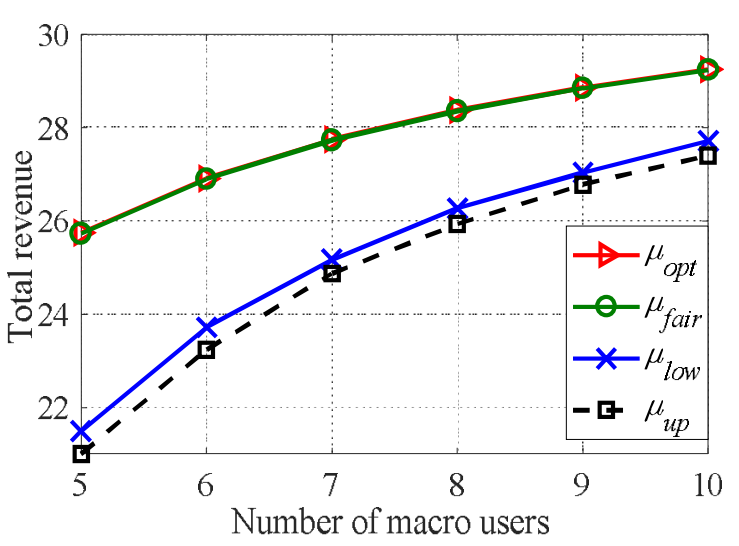

(b)

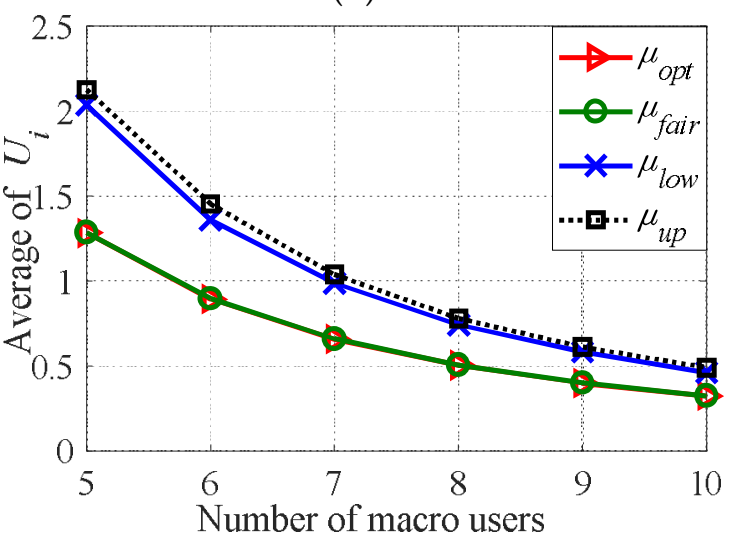

(d)

Figure 2. Impact of increasing the number of macro users on system performance with $Z_{i}=1$. (a) Satisfied factor of femto user $\left(\alpha^{*}\right)$; (b) Utility of FBS; (c) Average utility of macro users; (d) Total revenue of FBS.

It is clear that by increasing the number of MUs, the competition between them increases and this results in an increase in the revenue. Increasing revenue motivates FBS to improve its source further. Figure $2 \mathrm{c}$ depicts the fact that for maximizing its utility, the system prefers sharing more bandwidth rather than obtaining more revenue. As mentioned above, the system maximizes its revenue and utility by choosing $\mu_{o p t}$, so this raising revenue leads to further sharing of the bandwidth in comparison to the time when the system selects $\mu_{\text {low }}$ and $\mu_{u p}$.

Obviously, there is a trade-off between $U_{i}$ and UFBS i.e., promoting FBS and its femto user, reducing the importance of macro users. Although by choosing $\mu_{\text {low }}$ and $\mu_{u p}$ the bandwidth that FBS prefers to assign to MUs is less than the other method, the competition decreases by removing one MU; hence, the total revenue decreases. In this case, the shared bandwidth has a little difference with the case when the system selects $\mu_{\text {low }}$ and $\mu_{u p}$ but the overall revenue has more difference, so the average utility of MUs is higher than two other methods (Figure 2d).

\subsection{Assignment of Bandwidth Based on Different Decision Rules}

In this section, we investigate the way that communication system assigns its bandwidth according to different decision rules. In other words, the effect of different $\mu$ amounts on the extent of resources going to be allocated to each MU is discussed. Five macro users are placed near FBS, their priority factors are the same and the desired bandwidths for them are generated from a normal distribution with mean 16 and standard deviation 8. To demonstrate the detail of assignment, one snapshot is sufficient. Figure 3a depicts the accuracy of the point mentioned above which expresses when the system works with $\mu_{\text {fair }}$, the ratio of bandwidth allocation to the desired one for all macro users is the 
same if their priority factors are equal. In another aspect, when the system selects $\mu_{o p t}$, a macro user who needs less bandwidth is more satisfied. Also, macro users 5 and 2 show the case where an MU is less satisfied though it needs more bandwidth.

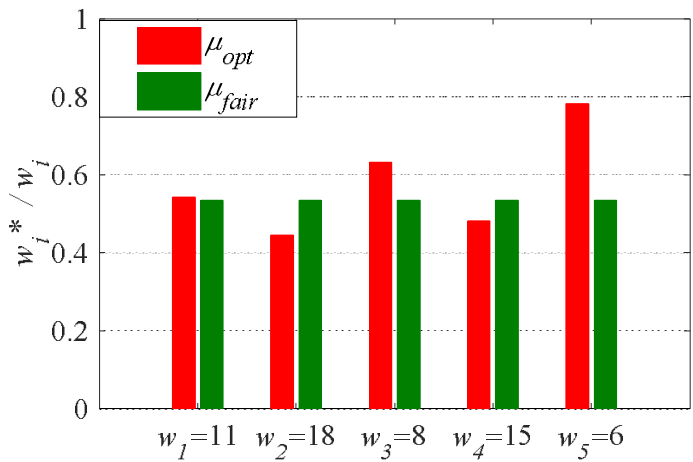

(a)

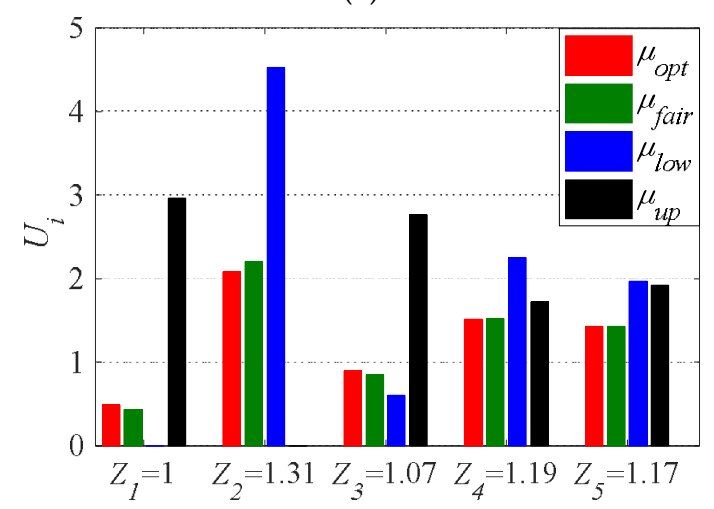

(c)

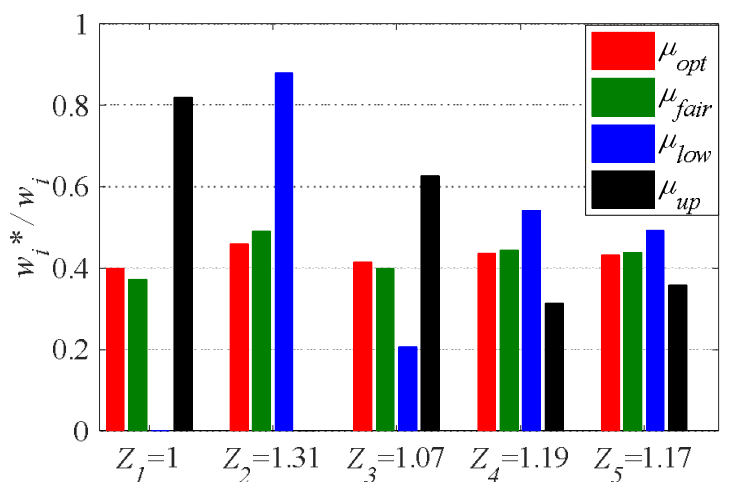

(b)

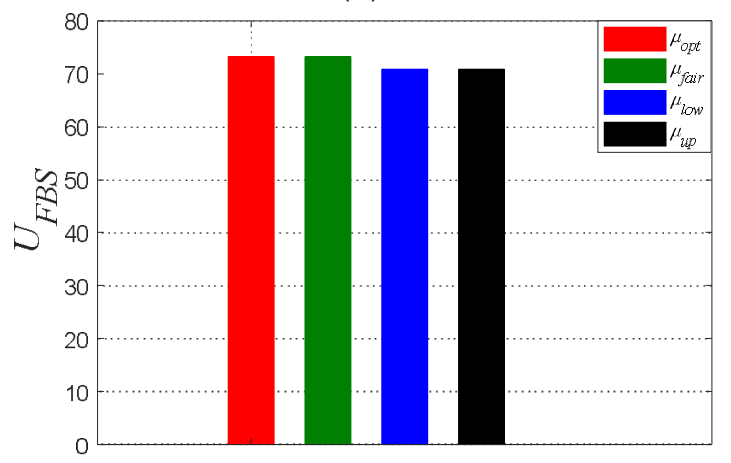

FU

(d)

Figure 3. Assignment of bandwidth to macro users (a) The ratio of the assigned bandwidth to desired one with different $w_{i} ;(\mathbf{b})$ The ratio of the assigned bandwidth to desired one with different priority; (c) Utility of each macro user; (d) Utility of FBS.

In order to analyze the influence of the priority parameter on the system performance, simulation parameters are adjusted as follows: assume that five macro users are distributed around one FBS and one of them has a priority factor equal to 1 . All macro users have the same bandwidth $\left(w_{i}=15\right)$ and the other priority factors are uniformly distributed between 1 and 1.4. In addition, one snapshot is sufficient to demonstrate the details of allocation.

Figure $3 \mathrm{~b}$ depicts that when the system works with $\mu_{\text {opt }}$ and $\mu_{\text {low }}$, it promotes MUs which have larger $w_{i} Z_{i}$, so the second macro user obtains maximum ratio of bandwidth allocation to the desired one. As we mentioned before, when the system selects $\mu_{\text {fare }}$, the system allocates bandwidth directly based on $Z_{i}$, so we see the second $M U$ has the maximum ratio of bandwidth allocation to the desired one, and it is higher than $\mu_{o p t}$. By choosing $\mu_{u p}$, the strategy changes completely and MU with minimum $w_{i} Z_{i}$ promotes; hence, unlike three previous methods, the system assigns maximum ratio of bandwidth allocation to the desired bandwidth to the first MU and deprives the second from allocation. Figure $3 c$ demonstrates the similar simulation result as Figure $3 b$ because of the same reason mentioned above. $\mu_{\text {opt }}$ has the maximum utility of FBS as provided in the previous section, next $\mu_{\text {fair }}, \mu_{\text {low }}$ and $\mu_{o p t}$ have the maximum utility, respectively which is represented in Figure $3 \mathrm{~d}$.

\subsection{Hybrid Access Model}

In this section, three models of access consisted of hybrid access, open access and closed access are assumed. Hybrid access is the proposed algorithm expressed with four above strategies of allocation. 
Without any revenue, FBS does not tend to share its source with macro users but if FBS is forced to share its bandwidth, FBS sees MUs as FUs, so in this case, the system assigns its resources equally. In other words, all macro and femto users have the same ratio of allocated bandwidth to the desired bandwidth. In closed access mode, the satisfaction factor of femto user is equal to 1 and the system does not share its bandwidth among other macro users.

The simulation parameters are considered as follows: three to 10 macro users are distributed around a FBS, and the desired bandwidths are uniformly distributed between 15 and 20 and the priority factor of them is equal to 1 . For better conclusion, 1000 snapshots are recorded and then their average is expressed. Figure 4a depicts that when the system works in hybrid access mode, the utility of FBS is the highest and goes up along with the number of macro users because the system can get more revenue if the number of macro users increases. In the closed access, the number of macro users does not affect the utility of FBS while in open access mode because all users have the same satisfaction factor, by increasing the number of macro users $\alpha$ decreases which causes a decrease in the utility of FBS. The satisfaction factor of FU in closed access is constant and equal to 1; in hybrid access mode increasing the number of macro users leads to increased revenue, so the system prefers to share more bandwidth and decreases $\alpha$ and finally, in open access mode increasing the number of macro users results in a decrease in value of $\alpha$ due to the same reason which is expressed above (Figure $4 \mathrm{~b}$ ).

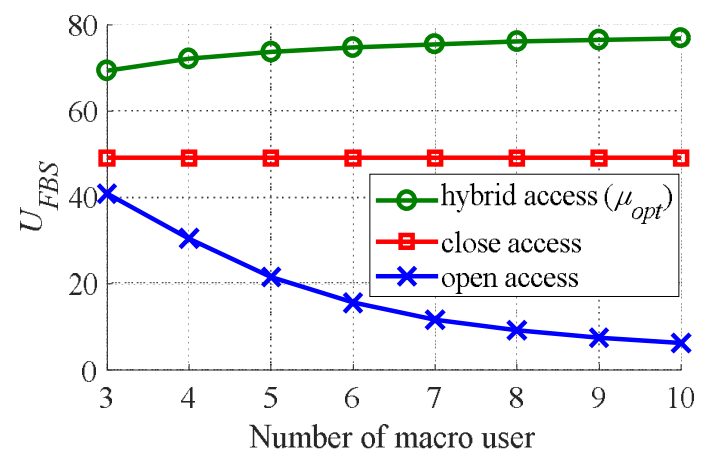

(a)

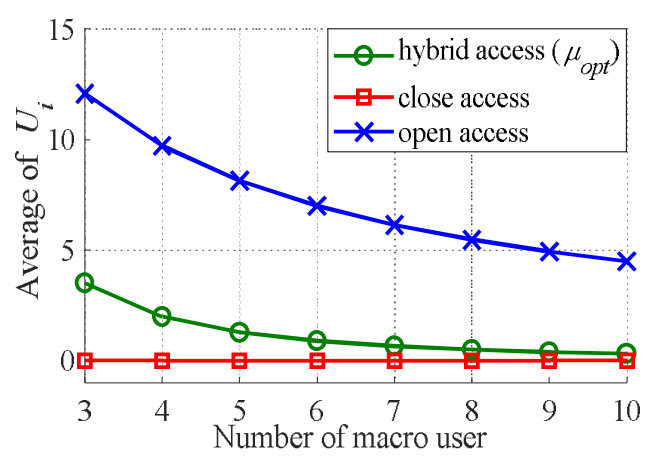

(c)

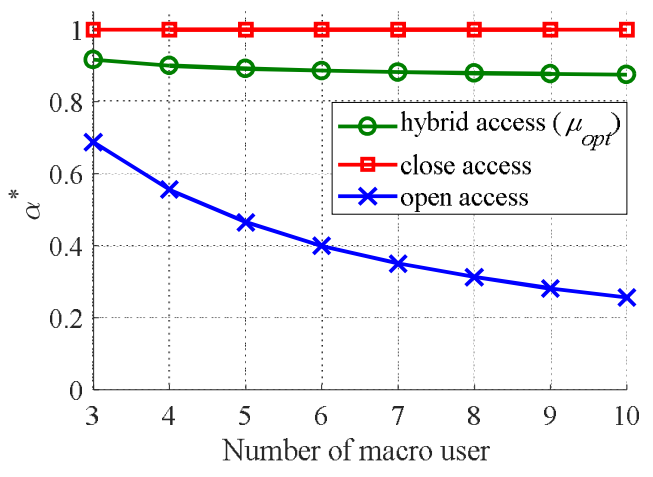

(b)

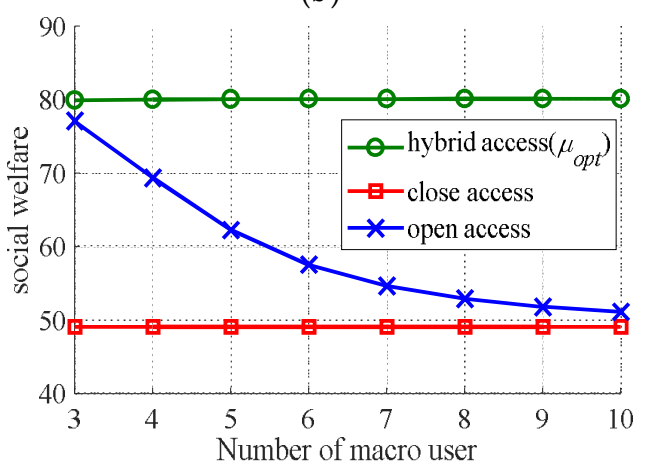

(d)

Figure 4. Hybrid, open and closed access comparison. (a) Utility of FBS; (b) Impact of increasing macro user in $\alpha^{*}$; (c) Average of $U_{i}$; (d) Social welfare.

Figure $4 \mathrm{c}$ demonstrates the average utility of macro user. Clearly, in open access, the average utility of MUs is the highest, so by increasing the number of macro users, the bandwidth is divided between macro users which decreases the utility of each MU. Also, a similar trend can be expected for open access. The social welfare which is expressed in Figure $4 \mathrm{~d}$ defines the summation of macro user's and FBS's utilities. Figure 4d shows that the social welfare in hybrid access mode is the highest and in open access mode, social welfare decreases if the number of macro users increases. Finally, closed access has constant and minimum social welfare. 


\subsection{The Impact of Interference on Hybrid Access}

In this section, we consider that there are some FBSs and one macro user placed adjacent to them while it is far from the MBS. In such a case, the victim macro user not only suffers from poor coverage of MBS but also interference signal that coming from other femtocells make the situation worse. Since the MU prefers to switch from MBS to one of the FBS, we need to consider the impact of different interference on our problem. When the MU connect to one FBS, the MBS signal make interference. Also other femtocells that are work in the same frequency and time can make interference for MU. Based on our assumption, The SINR for the victim macro user is expressed as:

$$
\operatorname{SINR}=\frac{P_{i} / L_{i}}{\sum_{j=1, j \neq i}^{k} P_{j} / L_{j}+P_{M} / L_{M}+\delta^{2}}
$$

where $P_{i}$ is the transmitted power from the connected $F B S_{i}$ and $L_{i}$ is the pathloss between $F B S_{i}$ and MU. $P_{j}$ and $L_{j}$ are the transmitted power from different FBSs and the pathloss between them and MU. $P_{M}$ and $L_{M}$ are the transmit power and path loss from MBS. $\delta^{2}$ is the noise power [33].

In order to investigate the impact of interference on our problem. We consider one MU is placed in the coverage range of FBSs and far from MBS. Figure 5 demonstrates that MU is moving on the arrow and the distance between MU and each FBS is changing. MU should make a decision which FBS can provide a better condition and then connect it. The larger SINR is better quality for MU obviously. Also, the larger SINR for FBS means FBS should spend less amount of power to guarantee a good quality of service for MU. We can conclude SINR has a good index to be considered as priority parameter $\left(Z_{i}\right)$. Based on the $\operatorname{SINR}, Z_{i}$ are normalized between $(0.7,1.4)$. In this regard, by moving MU toward the arrow, $Z_{i}$ changes and it leads to have different utility function amount. Since MU should connect the best FBS, it connects to the FBS that provide a larger $U_{i}$. The system parameters are summarized in Table 1 , in which $Z_{i}$ and $w_{i}$ are the priority and desired vector for entire MUs that are connected to $\mathrm{FBS}_{1}$ or $\mathrm{FBS}_{2}$. In the Figure 6a, the priority index is changed over the course of distance, and it is clear when the MU is closed to the connect FBS and has the most distance with the other FBS can obtain the most $Z_{i}$. Since the priority index is obtain based on SINR, and the system discerption doesn't have influence on $Z_{i}$, the both two priority curves are symmetric and exactly the same as each other. On the other hand, the MU prefers to connect to the FBS that has larger utility amount. Since the utility of victim MU depends on system parameters, the utility curve for each connection is different. Based on the Figure $6 \mathrm{~b}$, the MU prefers to switch to $\mathrm{FBS}_{2}$ when it has 10.66-meter distance from the starting point.

Table 1. Simulation parameters.

\begin{tabular}{cc}
\hline Parameter & Value \\
\hline Total amount of power for both FBS, MHz & 50 \\
$w_{i}$ for victim MUs & 18 \\
$w_{f}$ for MUs of $\mathrm{FBS}_{1}$ & 10 \\
$w_{i}$ for MUs of $\mathrm{FBS}_{1}$ & {$[10,12,15,8]$} \\
$Z_{i}$ for MUs of $\mathrm{FBS}_{1}$ & {$[1.1,1,1.2,1.4]$} \\
$w_{f}$ for MUs of $\mathrm{FBS}_{2}$ & 15 \\
$w_{i}$ for MUs of $\mathrm{FBS}_{2}$ & {$[13,13,12,9]$} \\
$Z_{i}$ for MUs of $\mathrm{FBS}_{2}$ & {$[1.1,1.2,1.3,1.2]$} \\
Distance from MBS, $\mathrm{m}$ & 700 \\
Path Loss $L_{M}, \mathrm{~dB}$ & $135+36 \log (d) ; \mathrm{d}$ in Km \\
Path Loss $L_{j}, \mathrm{~dB}$ & $38.5+20 \log (d) ; \mathrm{d}$ in $\mathrm{m}$ \\
$P_{M}$ & 50 \\
$P \_i$ & 1 \\
Thermal noise power $\mathrm{dBm} / \mathrm{Hz}$ & -174 \\
\hline
\end{tabular}




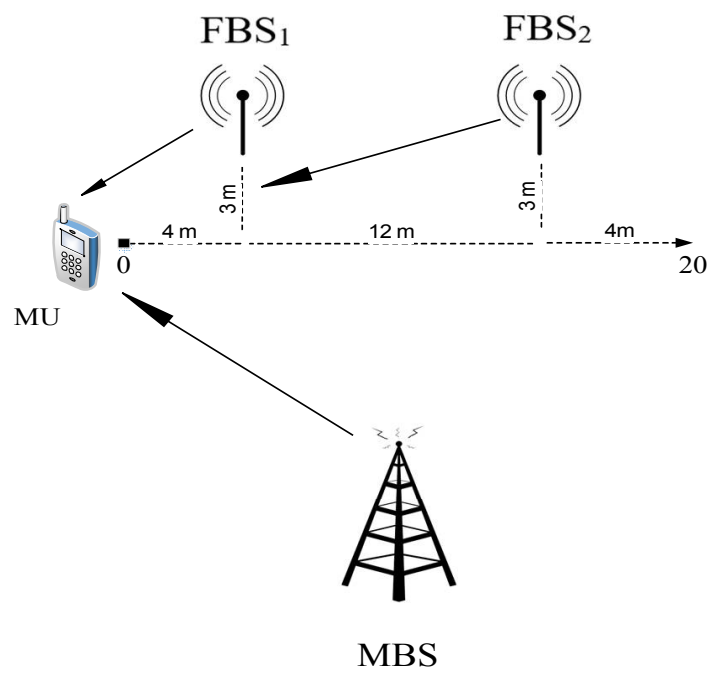

Figure 5. System description.

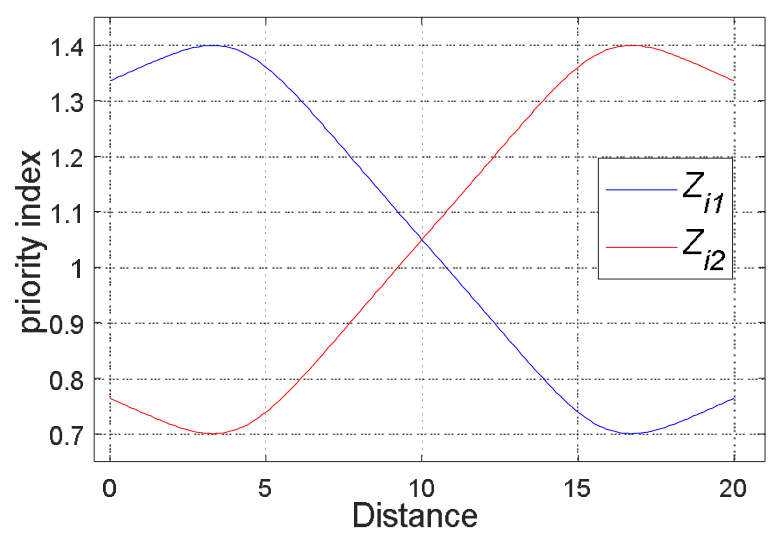

(a)

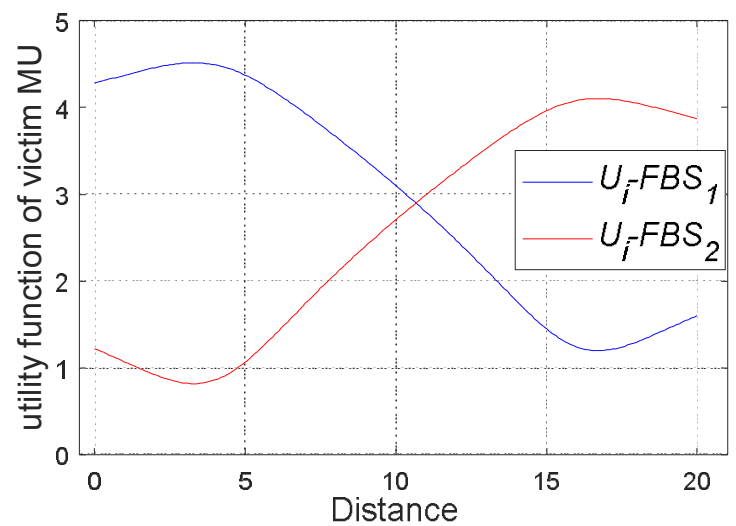

(b)

Figure 6. Priority index (a); utility function of the macro user (b).

\section{Conclusions}

In this paper, we proposed a Stackelberg game for optimal assignment of bandwidth to macro and femto users. The proposed method improves the efficiency of the femtocell communication network that can facilitate communications for a smarter electric grid. In our game, both macro- and femto users benefit from the proposed hybrid access schemes. To be more specific, macro users pay for their bandwidth. In this bandwidth allocation scheme, involves solving the KKT conditions for the underlying optimization problem to achieve optimal solution while ensuring the satisfaction of problem's constraints. Moreover, four strategies are studied for bandwidth allocation. Each strategy is designed to achieve a certain goal including; maximizing femtocell's utility, establishing fairness between macro users, disconnecting macro user with maximum desired bandwidth, promoting service for the macro user which requires minimum bandwidth and vice-versa. We have differentiated between macro users and designed our scheme so that the usage of some macro users is promoted to improve their satisfaction. Simulation results demonstrate that a system can choose the best strategy to rationally divide bandwidth between both macro users and femto users to optimize utility. In addition, numerical results illustrate that hybrid access mode leads to better social welfare compared with closed or open access modes. Specifically, in this case, macro users are willing to obtain more bandwidth while femto-base station is open to earning more revenue. Thus, this competition results in increasing revenue which motivates femto-base station to share more spectrum with macro users. 
Author Contributions: Mohammadi conceived the idea behind this research, designed the simulations and wrote the paper; Ghazizadeh and Dehghani performed the simulations and wrote some parts of the manuscript, respectively. All the authors analyzed the data and proofread the paper.

Funding: This work is not supported by any external funding.

Conflicts of Interest: The authors declare no conflict of interest.

\section{Appendix A}

Equation (10) of the KKT optimality conditions results in:

$$
\begin{gathered}
\lambda_{0}\left(w-\alpha w_{f}\right)\left[w_{x} \cdot \sum_{i=1}^{k} m_{i}-\mu \sum_{i=1}^{k} w_{i} Z_{i} m_{i}\right]=0 \\
\lambda_{i}\left(w_{x} \sum_{j=1}^{k} m_{j}-\left(w-\alpha w_{f}\right) \mathrm{m}_{j} Z_{j}\right) \geq 0, \lambda_{0}, \lambda_{i} \geq 0
\end{gathered}
$$

Solving the above equations leads to:

$$
w_{x} \sum_{j=1}^{k} m_{j}=\mu \sum_{j=1}^{k} w_{j} m_{j} Z_{j}, \lambda_{i}=0
$$

The optimal $m_{i}^{*}$ can be obtained as follows. Let us first equal $L\left(\alpha, \mathrm{m}_{i}, \lambda_{0}, \lambda_{i}\right)=0$ to zero. Then we can derive the following equations:

$$
\begin{gathered}
A \frac{X-m_{i}}{X^{2}} \mu w_{i} Z_{i}+B-Y \mu w_{i} Z_{i}=0 \\
\frac{\left(A X-Y X^{2}\right)}{A}+\frac{B X^{2}}{A \mu w_{i} Z_{i}}=m_{i}
\end{gathered}
$$

By adding equations related to each $\mathrm{MU}, \mathrm{X}$ is obtained as follows:

$$
\sum_{i=1}^{k} \frac{\left(A X-Y X^{2}\right)}{A}+\frac{B X^{2}}{A \mu w_{i} Z_{i}}=\sum_{i=1}^{k} m_{i}, X=\frac{(k-1) A}{k Y-B(D / \mu)},
$$

Since $X$ and $m_{\mathrm{i}}$ are dependent on $\lambda_{0}$, so $\lambda_{0}$ should be acquired from other parameters and can be removed from equations. In this case, by substituting (A5) into (A3), we can rewrite the constraints as:

$$
X w_{x}=\sum_{i=1}^{k}\left(\frac{\left(A X-Y X^{2}\right)}{A}+\frac{B X^{2}}{A \mu w_{i} Z_{i}}\right) \mu w_{i} Z_{i}
$$

By solving the above equation, $X$ is obtained as:

$$
X=\frac{A\left(w_{x}-\mu w_{x}\right)}{\left(B k-Y \mu w_{x}\right)}
$$

Given that we derived two closed from solutions form, we can calculate $\lambda_{0}$ by equating these equations:

$$
\lambda_{0}=\frac{D w_{x}-\mu\left(k-k^{2}+D w_{x}\right)}{\left(w-\alpha w_{f}\right) w_{x}\left(\mu^{2}+D w_{x}+\mu\left(-2 k+k^{2}-D w_{x}\right)\right)} .
$$




\section{References}

1. Bahrami, S.; Amini, M.H.; Shafie-khah, M.; Catalao, J.P. A Decentralized Electricity Market Scheme Enabling Demand Response Deployment. IEEE Trans. Power Syst. 2017. [CrossRef]

2. Bahrami, S.; Amini, M.H.; Shafie-khah, M.; Catalao, J.P. A Decentralized Renewable Generation Management and Demand Response in Power Distribution Networks. IEEE Trans. Sustain. Energy 2018. [CrossRef]

3. Amini, M.H.; Boroojeni, K.G.; Iyengar, S.; Pardalos, P.M.; Blaabjerg, F.; Madni, A.M. Sustainable Interdependent Networks: From Theory to Application; Springer: Berlin/Heidelberg, Germany, 2018.

4. Mohammadi, A.; Mehrtash, M.; Kargarian, A.; Barati, M. Tie-Line Characteristics based Partitioning for Distributed Optimization of Power Systems. arXiv 2018, arXiv:1805.09779.

5. Toulabi, M.; Bahrami, S.; Ranjbar, A.M. Application of Edge theorem for robust stability analysis of a power system with participating wind power plants in automatic generation control task. IET Renew. Power Gener. 2017, 11, 1049-1057. [CrossRef]

6. Bahrami, S.; Wong, V.W. Security-constrained unit commitment for ac-dc grids with generation and load uncertainty. IEEE Trans. Power Syst. 2017, 33, 2717-2732. [CrossRef]

7. Amini, M.H.; Karabasoglu, O. Optimal Operation of Interdependent Power Systems and Electrified Transportation Networks. Energies 2018, 11, 196. [CrossRef]

8. Toulabi, M.; Bahrami, S.; Ranjbar, A.M. An Input-to-State Stability Approach to Inertial Frequency Response Analysis of Doubly-Fed Induction Generator-Based Wind Turbines. IEEE Trans. Energy Convers. 2017, 32, 1418-1431. [CrossRef]

9. Kar, S.; Hug, G.; Mohammadi, J.; Moura, J.M. Distributed State Estimation and Energy Management in Smart Grids: A Consensus+ Innovations Approach. IEEE J. Sel. Top. Signal Process. 2014, 8, 1022-1038. [CrossRef]

10. Mohammadi, A.; Mehrtash, M.; Kargarian, A. Diagonal quadratic approximation for decentralized collaborative TSO+ DSO optimal power flow. IEEE Trans. Smart Grid 2018. [CrossRef]

11. Mehrtash, M.; Kargarian, A.; Mohammadi, A. Partition-based bus renumbering effect on interior point-based OPF solution. In Proceedings of the 2018 IEEE Texas Power and Energy Conference (TPEC), College Station, TX, USA, 8-9 February 2018; pp. 1-6.

12. Bahrami, S.; Amini, M.H. A Decentralized Trading Algorithm for an Electricity Market with Generation Uncertainty. arXiv 2017, arXiv:1705.02577. [CrossRef]

13. Shih, Y.-Y.; Pang, A.-C.; Tsai, M.-H.; Chai, C.-H. A rewarding framework for network resource sharing in co-channel hybrid access femtocell networks. IEEE Trans. Comput. 2015, 64, 3079-3090. [CrossRef]

14. Kang, X.; Zhang, R.; Motani, M. Price-based resource allocation for spectrum-sharing femtocell networks: A stackelberg game approach. IEEE J. Sel. Areas Commun. 2012, 30, 538-549. [CrossRef]

15. Chandrasekhar, V.; Andrews, J.G.; Muharemovic, T.; Shen, Z.; Gatherer, A. Power control in two-tier femtocell networks. IEEE Trans. Wirel. Commun. 2009, 8, 4316-4328. [CrossRef]

16. López-Pérez, D.; Valcarce, A.; de la Roche, G.; Zhang, J. OFDMA femtocells: A roadmap on interference avoidance. IEEE Commun. Mag. 2009, 47, 41-48. [CrossRef]

17. Chandrasekhar, V.; Andrews, J.G. Spectrum allocation in tiered cellular networks. IEEE Trans. Commun. 2009, 57, 3059-3068. [CrossRef]

18. Wu, Y.; Zhu, Q.; Huang, J.; Tsang, D.H. Revenue sharing based resource allocation for dynamic spectrum access networks. IEEE J. Sel. Areas Commun. 2014, 32, 2280-2296. [CrossRef]

19. Choi, D.; Monajemi, P.; Kang, S.; Villasenor, J. Dealing with loud neighbors: The benefits and tradeoffs of adaptive femtocell access. In Proceedings of the 2008 Global Telecommunications Conference, 2008 IEEE GLOBECOM, New Orleans, LA, USA, 30 November-4 December 2008; pp. 1-5.

20. Valcarce, A.; Lopez-Perez, D.; de la Roche, G.; Zhang, J. Limited access to OFDMA femtocells. In Proceedings of the 2009 IEEE 20th International Symposium on Personal, Indoor and Mobile Radio Communications, Tokyo, Japan, 13-16 September 2009; pp. 1-5.

21. Skoutas, D.N.; Makris, P.; Skianis, C. Optimized admission control scheme for coexisting femtocell, wireless and wireline networks. Telecommun. Syst. 2013, 53, 357-371. [CrossRef]

22. Xie, R.; Yu, F.R.; Ji, H.; Li, Y. Energy-efficient resource allocation for heterogeneous cognitive radio networks with femtocells. IEEE Trans. Wirel. Commun. 2012, 11, 3910-3920.

23. Duong, N.D.; Madhukumar, A.; Niyato, D. Stackelberg Bayesian game for power allocation in two-tier networks. IEEE Trans. Veh. Technol. 2016, 65, 2341-2354. [CrossRef] 
24. Liu, Y.; Chen, C.S.; Sung, C.W.; Singh, C. A Game Theoretic Distributed Algorithm for FeICIC Optimization in LTE-A HetNets. IEEE/ACM Trans. Netw. 2017, 25, 3500-3513. [CrossRef]

25. Yi, C.; Cai, J. Two-stage spectrum sharing with combinatorial auction and Stackelberg game in recall-based cognitive radio networks. IEEE Trans. Commun. 2014, 62, 3740-3752. [CrossRef]

26. Sundaresan, K.; Rangarajan, S. Efficient resource management in OFDMA femto cells. In Proceedings of the Tenth ACM International Symposium on Mobile Ad Hoc Networking and Computing, New Orleans, LA, USA, 18-21 May 2009; pp. 33-42.

27. Liang, Y.-S.; Chung, W.-H.; Ni, G.-K.; Chen, Y.; Zhang, H.; Kuo, S.-Y. Resource allocation with interference avoidance in OFDMA femtocell networks. IEEE Trans. Veh. Technol. 2012, 61, 2243-2255. [CrossRef]

28. Yu, J.; Han, S.; Li, X. A Robust Game-Based Algorithm for Downlink Joint Resource Allocation in Hierarchical OFDMA Femtocell Network System. IEEE Trans. Syst. Man Cybern. Syst. 2018. [CrossRef]

29. Tsiropoulou, E.E.; Vamvakas, P.; Papavassiliou, S. Supermodular game-based distributed joint uplink power and rate allocation in two-tier femtocell networks. IEEE Trans. Mob. Comput. 2017, 16, 2656-2667. [CrossRef]

30. Zhu, K.; Hossain, E.; Anpalagan, A. Downlink power control in two-tier cellular OFDMA networks under uncertainties: A robust Stackelberg game. IEEE Trans. Commun. 2015, 63, 520-535. [CrossRef]

31. Wang, B.; Wu, Y.; Liu, K.R. Game theory for cognitive radio networks: An overview. Comput. Netw. 2010, 54, 2537-2561. [CrossRef]

32. Zhang, N.; Cheng, N.; Lu, N.; Zhou, H.; Mark, J.W.; Shen, X. Risk-aware cooperative spectrum access for multi-channel cognitive radio networks. IEEE J. Sel. Areas Commun. 2014, 32, 516-527. [CrossRef]

33. Hamouda, S.; Zitoun, M.; Tabbane, S. Win-win relationship between macrocell and femtocells for spectrum sharing in LTE-A. IET Commun. 2014, 8, 1109-1116. [CrossRef]

34. Mohammadi, A.; Dehghani, M.J. Spectrum allocation using fuzzy logic with optimal power in wireless network. In Proceedings of the 2014 4th International eConference on Computer and Knowledge Engineering (ICCKE), Mashhad, Iran, 29-30 October 2014; pp. 532-536.

35. Chen, Y.; Zhang, J.; Zhang, Q. Utility-aware refunding framework for hybrid access femtocell network. IEEE Trans. Wirel. Commun. 2012, 11, 1688-1697. [CrossRef]

36. Zhang, J.; Zhang, Q. Stackelberg game for utility-based cooperative cognitiveradio networks. In Proceedings of the Tenth ACM International Symposium on Mobile Ad Hoc Networking and Computing, New Orleans, LA, USA, 18-21 May 2009; pp. 23-32. 